\title{
Green nanovectors for phytodrug delivery: in-depth structural and morphological characterization
}

\author{
Ilaria Clemente ${ }^{a, b^{*}}$, Sara Falsinic, Emanuela Di Cola ${ }^{d}$, Giulia C. Fadda ${ }^{e f}$, Cristina Gonnellic, \\ Francesco Spinozzi ${ }^{g}$, Maria Bacia-Verloop ${ }^{h}$, Isabelle Grillo ${ }^{i}$, Sandra Ristori ${ }^{b^{*}}$
}

a) Department of Biotechnology, Chemistry and Pharmacy, University of Siena, Siena, 53100, Italy b) Department of Chemistry “Ugo Schiff” \& CSGI, University of Florence, Sesto Fiorentino, 50019, Italy c) Department of Biology, University of Florence, Florence, 50121, Italy

d) Dipartimento di Biotecnologie Mediche e Medicina Traslazionale, Università degli Studi di Milano, 20900 Segrate, Italy;

e) Laboratoire Léon Brillouin, CNRS, CEA, Université Paris-Saclay, 91191 Gif-sur-Yvette Cedex, France f) Université Paris 13, UFR SMBH, 93017 Bobigny, France

g) Department of Life and Environmental Sciences, Polytechnic University of Marche, 60131, Ancona, Italy

h) CNRS, UMR 5075, Institut de Biologie Structurale, 38027 Grenoble, France

i) Institut Max von Laue-Paul Langevin, 38042 Grenoble Cedex 9, France

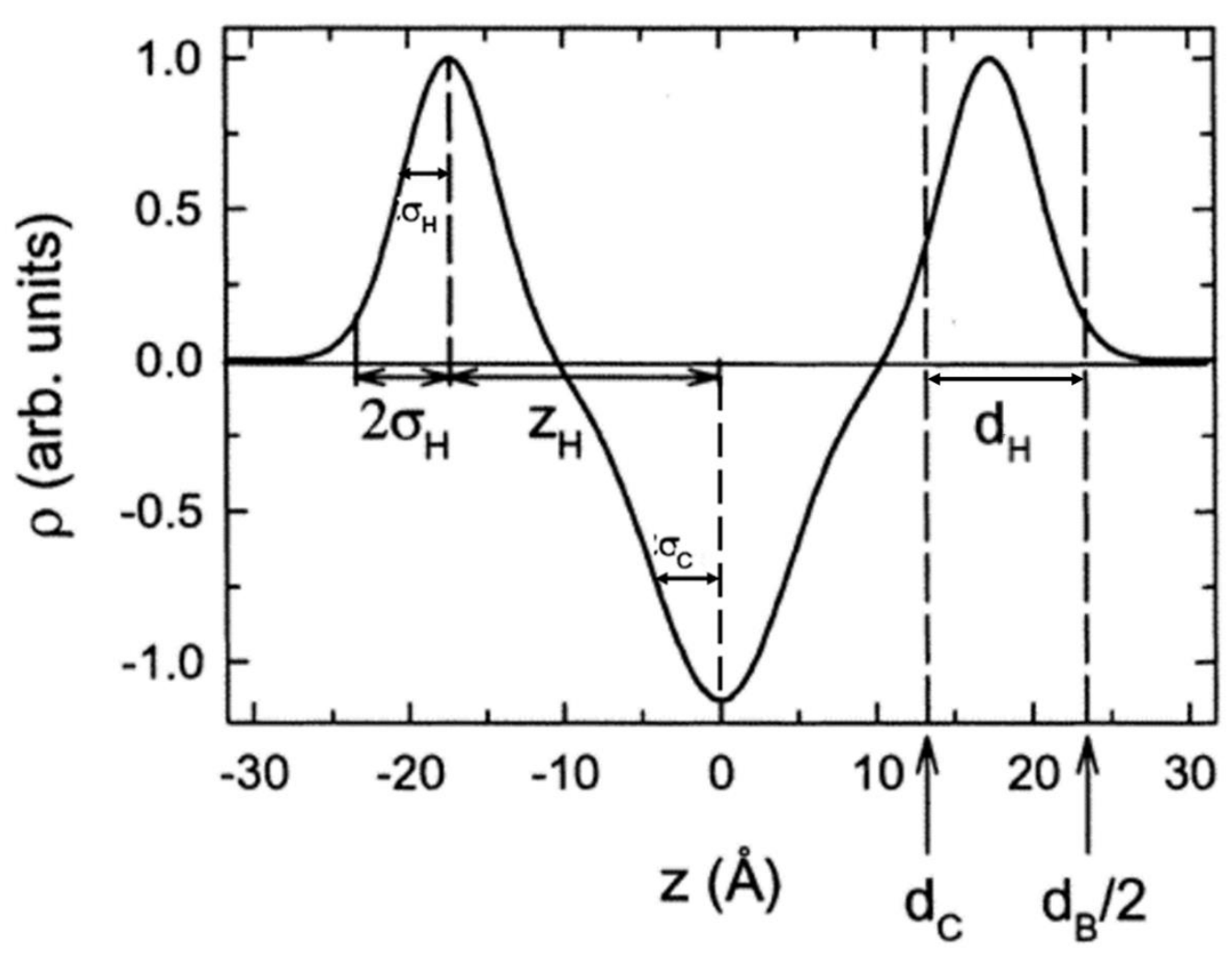

Fig. S1. Scheme of the model used to represent the electron density profile as sum of three Gaussians representing the polar head groups at $\pm \mathrm{Z}_{\mathrm{H}}$ and the terminal methyl group at the center of the bilayer (see text for more details). Reprinted with slight modification from Pabst et al. Langmuir 2003, 19, 1716-1722 
Equations used for the fitting of SAXS profiles of the PE sample series. The structural arrangement is modeled as hexagonal mesophases built up from large vesicles surrounded by a three-electron densities domain symmetric bilayer. See text for parameter definition and further details

$$
\begin{aligned}
& I(q)=\frac{\varphi_{h b}}{16 \pi\left(R_{2}+R_{3}\right)} \sum_{k_{1}, k_{2}=0}^{1} \frac{\Gamma\left(s<R_{0}>-2+k_{1}+k_{2}\right) s^{2-k_{1}-k_{2}}}{\Gamma\left(s<R_{0}>\right)}\left[\Im\left(f_{k_{1}}\right) \mathfrak{I}\left(f_{k_{2}}\right)+\mathfrak{R}\left(f_{k_{1}}\right) \mathfrak{R}\left(f_{k_{2}}\right)\right]+
\end{aligned}
$$

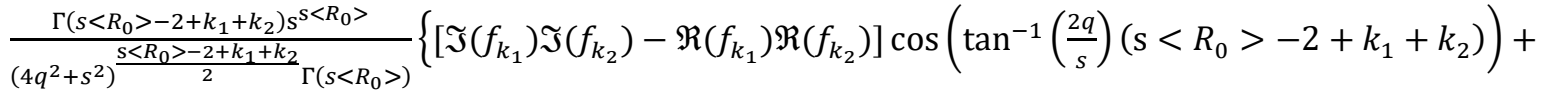

$$
\begin{aligned}
& \left.\left[\Re\left(f_{k_{1}}\right) \mathfrak{I}\left(f_{k_{2}}\right)+\mathfrak{J}\left(f_{k_{1}}\right)\left(f_{k_{2}}\right)\right] \sin \left(\tan ^{-1}\left(\frac{2 q}{s}\right)\left(\mathrm{s}<R_{0}>-2+k_{1}+k_{2}\right)\right)\right\}+\frac{\varphi_{h b} \varphi_{c}<R_{0}>}{6 q R_{c}^{2}\left(R_{2}+R_{3}\right)}\left[F_{c}(q)\right]^{2}\left\langle S_{c c}(\mathbf{q})\right\rangle_{\left\{\alpha_{q}\right\}}(\mathrm{S} 1)
\end{aligned}
$$

where $s=1 /\left(<R_{0}>\xi_{R_{0}}^{2}\right)$. Real and imaginary parts of the complex factors $f_{k}$ are found to be

$$
\begin{aligned}
& \Re\left(f_{0}\right)=\frac{4 \pi}{q^{3}}\left(\left(\rho_{4}-\rho_{1}\right)+\left(\rho_{3}-\rho_{2}\right)(\cos (c q)+c q \sin (c q)-\cos (d q)-d q \sin (d q))+\left(\rho_{2}-\rho_{1}\right)(\cos (b q)+\right. \\
& \left.\left.b q \sin (b q)-\cos \left(R_{1} q\right)-R_{1} q \sin \left(R_{1} q\right)\right)+\left(\rho_{1}-\rho_{0}\right)(\cos (t q)+t q \sin (t q))\right) \\
& \Im\left(f_{0}\right)=-\frac{4 \pi}{q^{3}}\left(\left(\rho_{3}-\rho_{2}\right)(\sin (d q)-d q \cos (d q)-\sin (c q)+c q \cos (c q))+\left(\rho_{2}-\rho_{1}\right)(-\sin (b q)+b q \cos (b q)+\right. \\
& \left.\left.\sin \left(R_{1} q\right)-R_{1} q \cos \left(R_{1} q\right)\right)+\left(\rho_{1}-\rho_{0}\right)(-\sin (t q)+t q \cos (t q))\right) \\
& \Re\left(f_{1}\right)=-\frac{4 \pi}{q^{2}}\left(\left(\rho_{3}-\rho_{2}\right)(\sin (d q)-\sin (c q))+\left(\rho_{2}-\rho_{1}\right)\left(\sin \left(R_{1} q\right)-\sin (b q)\right)-\sin (t q)\left(\rho_{1}-\rho_{0}\right)\right) \\
& \Im\left(f_{1}\right)=-\frac{4 \pi}{q^{2}}\left(\left(\rho_{4}-\rho_{1}\right)+\left(\rho_{3}-\rho_{2}\right)(\cos (c q)-\cos (d q))+\left(\rho_{2}-\rho_{1}\right)\left(\cos (b q)-\cos \left(R_{1} q\right)\right)+\cos (t q)\left(\rho_{1}-\rho_{0}\right)\right) \\
& F_{c}(q)=2 \pi\left(\left(\rho_{c, 3}-\rho_{c, 2}\right) R_{c, 3}^{2} \frac{\mathrm{J}_{1}\left(q R_{c, 3}\right)}{q R_{c, 3}}+\left(\rho_{c, 2}-\tilde{\mathrm{n}}_{c, 1}\right)\left(R_{c, 2}+R_{c, 3}\right)^{2} \frac{\mathrm{J}_{1}\left(q\left(R_{c, 2}+R_{c, 3}\right)\right)}{q\left(R_{c, 2}+R_{c, 3}\right)}+\left(\rho_{c, 1}-\rho_{4}\right) R_{c}^{2} \frac{\mathrm{J}_{1}\left(q R_{c}\right)}{q R_{c}}\right)
\end{aligned}
$$

where $\mathrm{J}_{1}(x)$ is the first order Bessel function 


\begin{tabular}{|c|c|c|c|c|c|}
\hline Parameter & Note & Unit & EPE & IPE & NPE \\
\hline$<R_{0,1}>$ & $\mathrm{a}$ & $(\AA)$ & $5000 \pm 600$ & $4900 \pm 600$ & $5000 \pm 300$ \\
\hline$<R_{0,2}>$ & a & $(\AA)$ & $3600 \pm 200$ & $4100 \pm 400$ & $3500 \pm 100$ \\
\hline$<R_{0,3}>$ & a & $(\AA)$ & $640 \pm 30$ & $980 \pm 40$ & $580 \pm 20$ \\
\hline$\xi_{R_{0,1}}$ & & & $0.7 \pm 0.3$ & $0.6 \pm 0.1$ & $0.7 \pm 0.2$ \\
\hline$\xi_{R_{02}}$ & & & $0.700 \pm 0.001$ & $0.68 \pm 0.05$ & $0.700 \pm 0.005$ \\
\hline$\xi_{R_{0,3}}$ & & & $0.70 \pm 0.01$ & $0.70 \pm 0.02$ & $0.700 \pm 0.005$ \\
\hline$R_{1,1}$ & $\mathrm{~b}$ & $(\AA)$ & $6.4 \pm 0.2$ & $8 \pm 2$ & $6.4 \pm 0.4$ \\
\hline$R_{1,2}$ & $\mathrm{~b}$ & $(\AA)$ & $7 \pm 2$ & $9 \pm 2$ & $9 \pm 1$ \\
\hline$R_{1,3}^{1,2}$ & $\mathrm{~b}$ & $(\AA)$ & $14.75 \pm 0.1$ & $10 \pm 2$ & $11 \pm 1$ \\
\hline$R_{2,1}^{1,0}$ & c & $(\AA)$ & $8 \pm 1$ & $8.1 \pm 0.4$ & $8.0 \pm 0.5$ \\
\hline$R_{2,2}^{2,1}$ & $\mathrm{c}$ & $(\AA)$ & $19.7 \pm 0.3$ & $20.0 \pm 0.3$ & $18.4 \pm 0.7$ \\
\hline$R_{2,3}^{2,2}$ & $\mathrm{c}$ & $(\AA)$ & $11 \pm 2$ & $17 \pm 2$ & $14 \pm 2$ \\
\hline$R_{3,1}^{2,}$ & d & $(\AA)$ & $3.7 \pm 0.6$ & $2.02 \pm 0.1$ & $2.2 \pm 0.3$ \\
\hline$R_{3,2}$ & d & (A) & $3.5 \pm 0.2$ & $3.6 \pm 0.7$ & $3.6 \pm 0.2$ \\
\hline$R_{3,3}$ & d & $(\AA)$ & $3.4 \pm 0.2$ & $3.9 \pm 0.4$ & $4.0 \pm 0.5$ \\
\hline$\rho_{1,1}$ & $\mathrm{e}$ & $\left(\mathrm{e}^{-3}\right)$ & $0.41 \pm 0.01$ & $0.414 \pm 0.002$ & $0.414 \pm 0.01$ \\
\hline$\rho_{1,2}$ & e & $\left(\mathrm{e}^{-3}\right)$ & $0.40 \pm 0.01$ & $0.42 \pm 0.02$ & $0.389 \pm 0.01$ \\
\hline$\rho_{1,3}$ & e & $\left(\mathrm{e}^{-3}\right)$ & $0.419 \pm 0.01$ & $0.392 \pm 0.01$ & $0.416 \pm 0.03$ \\
\hline$\rho_{2,1}$ & f & $\left(\mathrm{e}^{-3}\right)$ & $0.285 \pm 0.01$ & $0.300 \pm 0.01$ & $0.280 \pm 0.04$ \\
\hline$\rho_{2,2}$ & f & $\left(e^{-3}\right)$ & $0.291 \pm 0.01$ & $0.303 \pm 0.01$ & $0.2812 \pm 0.001$ \\
\hline$\rho_{2,3}$ & $\mathrm{f}$ & $\left(\mathrm{e}^{-3}\right)$ & $0.28 \pm 0.02$ & $0.29 \pm 0.01$ & $0.300 \pm 0.01$ \\
\hline$\rho_{3,1}$ & $\mathrm{~g}$ & $\left(e^{-3}\right)$ & $0.24 \pm 0.02$ & $0.30 \pm 0.02$ & $0.23 \pm 0.01$ \\
\hline$\rho_{3,2}$ & $\mathrm{~g}$ & $\left(\mathrm{e}^{-3}\right)$ & $0.21 \pm 0.03$ & $0.22 \pm 0.02$ & $0.20 \pm 0.03$ \\
\hline$\rho_{3,3}$ & $\mathrm{~g}$ & $\left(\mathrm{e}^{-3}\right)$ & $0.20 \pm 0.04$ & $0.21 \pm 0.02$ & $0.25 \pm 0.03$ \\
\hline$\rho_{4,2}$ & $\mathrm{~h}$ & $\left(e \AA^{-3}\right)$ & $0.3269 \pm 0.01$ & $0.326 \pm 0.03$ & $0.325 \pm 0.04$ \\
\hline$\rho_{4,3}$ & $\mathrm{~h}$ & $\left(\mathrm{e}^{-3}\right)$ & $0.297 \pm 0.01$ & $0.327 \pm 0.03$ & $0.312 \pm 0.03$ \\
\hline$\varphi_{h b, 1}$ & $\mathrm{i}$ & & $0.02 \pm 0.03$ & $0.010 \pm 0.003$ & $0.020 \pm 0.004$ \\
\hline$\varphi_{h b, 2}$ & $\mathrm{i}$ & & $0.014 \pm 0.002$ & $0.003 \pm 0.003$ & $0.016 \pm 0.004$ \\
\hline$\varphi_{h b, 3}$ & $\mathrm{i}$ & & $0.0005 \pm 0.0007$ & $0.003 \pm 0.002$ & $0.005 \pm 0.002$ \\
\hline$R_{c, 1,2}$ & $\mathrm{~b}$ & $(\AA)$ & $6.001 \pm 0.04$ & $6.00 \pm 0.1$ & $6.000 \pm 0.01$ \\
\hline$R_{c, 1,3}$ & $\mathrm{~b}$ & $(\AA)$ & $6.2 \pm 0.1$ & $6.0 \pm 0.5$ & $6.00 \pm 0.2$ \\
\hline$R_{c, 2,2}$ & $\mathrm{c}$ & $(\AA)$ & $10.0 \pm 0.8$ & $8 \pm 2$ & $8.010 \pm 0.1$ \\
\hline$R_{c, 2,3}$ & $\mathrm{c}$ & $(\AA)$ & $8 \pm 3$ & $20 \pm 5$ & $17 \pm 1$ \\
\hline$R_{c, 3,2}$ & d & $(\AA)$ & $2.1 \pm 1$ & $2.0 \pm 0.9$ & $2.0 \pm 0.3$ \\
\hline$R_{c, 3,3}$ & $\mathrm{~d}$ & $(\AA)$ & $2.2 \pm 0.9$ & $3.8 \pm 0.2$ & $3.9 \pm 0.8$ \\
\hline$\rho_{c, 1,2}$ & e & $\left(e^{-3}\right)$ & $0.370 \pm 0.02$ & $0.370 \pm 0.01$ & $0.370 \pm 0.01$ \\
\hline$\rho_{c, 1,3}$ & e & $\left(\mathrm{e}^{-3}\right)$ & $0.40 \pm 0.01$ & $0.370 \pm 0.01$ & $0.370 \pm 0.01$ \\
\hline$\rho_{c, 2,2}$ & f & $\left(e \AA^{-3}\right)$ & $0.304 \pm 0.01$ & $0.283 \pm 0.01$ & $0.297 \pm 0.03$ \\
\hline$\rho_{c, 2,3}$ & $\mathrm{f}$ & $\left(\mathrm{e}^{\circ} \AA^{-3}\right)$ & $0.30 \pm 0.02$ & $0.300 \pm 0.01$ & $0.280 \pm 0.01$ \\
\hline$\rho_{c, 3,2}$ & $\mathrm{~g}$ & $\left(e^{-3}\right)$ & $0.28 \pm 0.01$ & $0.22 \pm 0.03$ & $0.25 \pm 0.03$ \\
\hline$\rho_{c, 3,3}$ & $\mathrm{~g}$ & $\left(e^{-3}\right)$ & $0.24 \pm 0.02$ & $0.26 \pm 0.03$ & $0.20 \pm 0.03$ \\
\hline$g_{a, 2}$ & $\mathrm{j}$ & & $0.00 \pm 0.02$ & $0.00 \pm 0.01$ & $0.00 \pm 0.01$ \\
\hline$g_{a, 3}$ & $\mathrm{j}$ & & $0.023 \pm 0.005$ & $0.026 \pm 0.002$ & $0.030 \pm 0.001$ \\
\hline$M / M_{\max _{2}}$ & $\mathrm{k}$ & & $0.6 \pm 0.3$ & $0.6 \pm 0.4$ & $0.9 \pm 0.3$ \\
\hline$M / M_{\max _{3}}$ & $\mathrm{k}$ & & $0.99 \pm 0.04$ & $1.0 \pm 0.1$ & $1.0 \pm 0.4$ \\
\hline$B$ & 1 & $\left(10^{-5} \mathrm{~mm}^{-1}\right)$ & $13.1 \pm 0.5$ & $8.5 \pm 0.5$ & $17.2 \pm 0.7$ \\
\hline
\end{tabular}

Table S1. Best fit parameters of the PE SAXS curves obtained by Genfit adopting the hexosome model (equation 5). Validity ranges of fitting parameters: ${ }^{\mathrm{a}} 400 \div 5000 ;{ }^{\mathrm{b}} 5 \div 15 ;{ }^{\mathrm{c}} 8 \div 20 ;{ }^{\mathrm{d}} 2 \div 4 ;{ }^{\mathrm{e}} 0.37 \div 0.42 ;{ }^{\mathrm{f}} 0.28 \div 0.32$; ${ }^{\mathrm{g}} 0.20 \div 0.28 ;{ }^{\mathrm{h}} 0.28 \div 0.33 ;{ }^{\mathrm{I}} 0 \div 0.2,{ }^{\mathrm{j}} 10^{-10} \div 0.03 ;{ }^{\mathrm{k}} 0 \div 1 ;{ }^{\mathrm{l}} 0 \div 30$. $B$ is a flat background. 

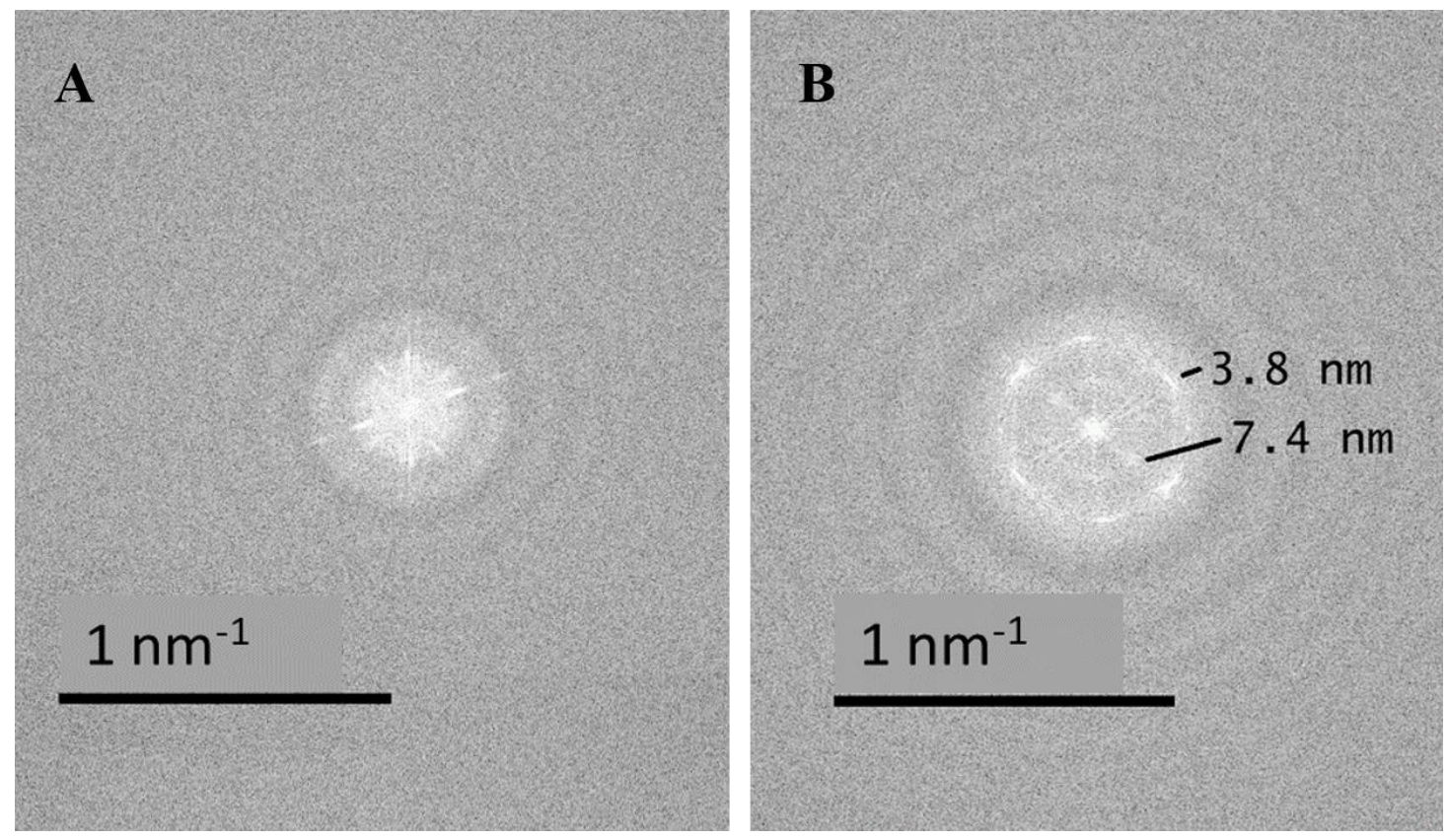

Fig S2. FFT of Cryo-TEM micrographs of PC (A) samples showing lamellar vesicles and PE (B) samples showing hexagonal arrangement. 